\title{
COST OPTIMIZATION OF PRODUCT FAMILIES USING SOLUTION SPACES
}

\author{
S. Rötzer ${ }^{1, \otimes}$, D. Thoma ${ }^{2}$ and M. Zimmermann ${ }^{1}$ \\ ${ }^{1}$ Technical University of Munich, Germany, ${ }^{2}$ ID-Consult GmbH, Germany \\ $\triangle$ sebastian.roetzer@tum.de
}

\section{Abstract}

Maximizing the number of shared components does not always produce cost-optimal product families. Shared components can yield economies of scale due to reuse of components, but also increases material cost due to over dimensioning. In this paper, we present an approach to identify a cost-optimal design for product families. It consists of two steps: (1) identifying a scheme to share components in the product family and (2) finding the cost optimal design for the product family.

Keywords: complex systems, systems engineering (SE), cost optimisation, solution space engineering, product families

\section{Introduction}

Experience at a consultancy firm specialized in modular platform development has shown that many companies struggle to cope with the variety in their complex product portfolios. They have to find the optimal fit between external and internal variety. While external variety is concerned with offering the right products to the market, internal variety includes the question of optimal standardization. In some cases, different products or the components, which fulfil the same requirements, can be standardized. In other cases, designers need to over-dimension components in order to standardize them.

On the one hand, standardization can yield economies of scale due to the reuse of components, one the other hand it comes along with increased material cost due to over-dimensioning.

This leads to the question of cost optimal commonality, represented by two questions from industry projects:

- For a product family of automotive ESP (electronic stability program) systems: How many variants of the electric motor should be developed to cover a spectrum of requested torque and power most cost efficiently?

- For a product family of solar inverters: How many power steps of the hardware inverter should be produced in order to minimize cost? (Further power steps are offered by applying control software)

In practice, technical consultants try to answer this question by developing scenarios, evaluating them and choosing the best. Refining the scenarios iteratively can lead to satisfactory results. However, this approach cannot guarantee an optimal solution. This can lead to a high iteration efforts and lacking precision, if scenarios are only evaluated qualitatively. Furthermore, technical consultants observe that 
companies tend to request quantitatively provable results. This demands for new methods to optimize product portfolios or product families numerically. In the next section, we present existing approaches for quantitative optimization of product families.

\section{Related research}

A product family is a group of products that share a common platform (Simpson et al., 2001, p. 3). Distinguish two types of product families due to the way the variants are derived. In scale-based product families variants are created by scaling the product along one or multiple parameters. In module-based product families variants are created by exchanging modules to alter the functionality of the variants. This paper focuses on scale-based product families. Choosing the right commonality of components prevails in this field. Optimization of scale-based product families involves two fundamental tasks (Simpson, 2004):

1. Deciding which will be the platform and scaling variables of the product family

2. Choosing the optimal values for the platform and scaling variables

In many methods the two steps are carried out sequentially in order to reduce the computational effort. Simpson (2006) provides an overview over many two-step approaches. However, choosing the platform a-priori may lead to suboptimal solutions (Chowdhury et al., 2011, p. 2). Therefore, one-step approaches have been developed, in which the choice of the platform and the variable values is optimised simultaneously. The reduce the computational effort in these approaches some authors used the simplification that components would either shared between all products or none (Simpson and D'Souza, 2004; Fellini et al., 2004). This simplification is overcome e.g. by Khajavirad and Michalek (2008) who present a method for generalized commonality, allowing components to be also shared among subsets of the product family. Yet, calculating all different possibilities to share components between product then becomes prohibitively expensive for larger product families (Zapico et al., 2015, p. 8). Chowdhury et al. (2010) present a one-step approach for product family optimization using a reduced commonality matrix to the computational cost. Nevertheless, this approach has a critical computation time for a product family of 7 products.

Eichstetter et al. (2015) propose a method for product family design using solution spaces to reduce the computational effort. Zimmermann et al. (2017) laid the basis for, as we call it, Solution Space Engineering approach. It breaks down requirements from system level to sub-system and component level using quantitative models. By fulfilling the requirements on component level, the approach makes sure that the overall system requirements are fulfilled. Thus, it can be seen as a quantitative V-model approach. It calculates and evaluates the system responses of different designs. A design is defined as a set of design variables. Those sets of design variables are Monte Carlo sampled uniformly between certain borders - the design space. Designs which fulfil all requirements are called good designs, the ones, which violate one or more requirements are called bad designs. The set of all good designs forms the solution space. Putting a rectangular box in the solution space allows to decouple the design variables. This rectangular solution space consists of intervals for each design variable. All combinations of those design variables will fulfil all requirements, as long as they stay within the rectangular solution box. This means, within the respective interval, every design variable can be chosen independently without creating overall bad solutions. By considering this, we can optimize every variable independently for the product family and overcome the previously presented problem of combinatorics.

Assuming that large boxes provide a greater potential to find overlaps between the boxes of the different product variants, Eichstetter et al. (2015) apply an algorithm to maximize the size of the rectangular boxes. After a rectangular box has been determined, an algorithm searches the minimum number of design variable values needed to cover all product variants. For this purpose, the algorithm starts by trying out the lowest theoretical variety and adds further variable values until every product variant is assigned a value. In this procedure, not every possible combination needs to be calculated, so that the computational effort is reduced.

However, it is uncertain whether maximum commonality equals cost-optimal commonality. The costoptimum of the single product without sharing components normally differs from solutions that provide increased component sharing. The optimality of a solution can be evaluated using different criteria such 
a time, quality or risk. According to Skirde et al. (2015) these target quantities can all be reduced to cost. Hence, minimizing the cost of the product family is a proxy to the conflict of objectives.

\section{Method: Cost optimization of product families}

The proposed method evaluates both: fulfilling the requirements and cost optimality. It consists of three steps. Steps 1 and 2 ensure the that the sets fulfil all requirements and are based on Eichstetter et al. (2015). Step 3 determines the optimal commonality. Figure 1 depicts the three steps of the method.

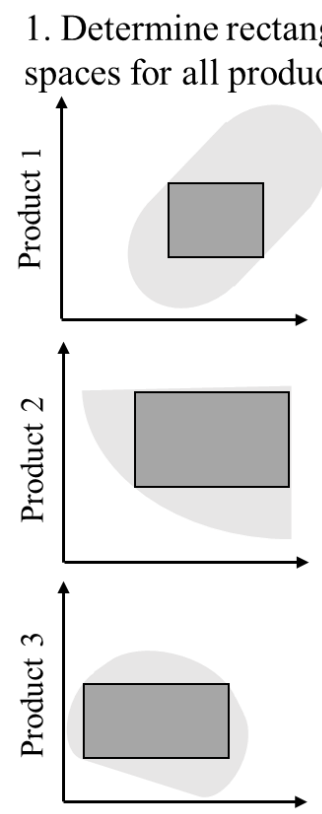

2. Identify overlaps of solution spaces

lar solution

ariants
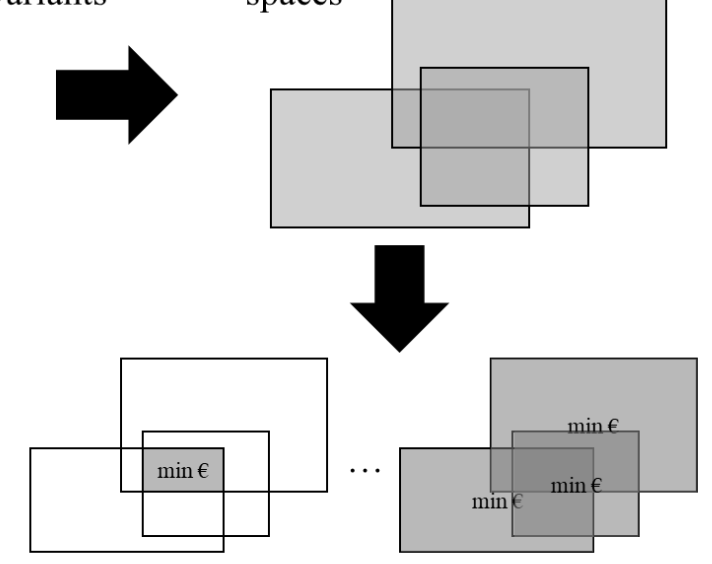

3. Optimize design variable values for every possible combination to design a product family with respect to the cost of the product family

Figure 1. Method: Cost optimization of product families

1. Determine rectangular solution spaces for all product variants, i.e. sets of design variables, which fulfil the requirements of the corresponding product variant

In order to calculate the solution spaces, we need quantitative models of the system at hand. The models map the design variables, which the designer can change, to the objective quantities, which represent the system performance. Requirements are typically formulated with respect to the objective quantities and we need to know their quantitative values for each product as well. Once we have all this information, we calculate solution spaces for each product.

2. Identify overlaps of solution spaces, i.e. the potential for standardization from technical perspective

By showing solution spaces in one diagram, we can identify areas, where commonality by standardization is technically possible. Within the overlapping areas, design variable values can be chosen in a way that they can be used commonly for multiple variants. This means, a component can be shared between two or more products because it fulfils the requirements of those products.

3. Optimize design variable values for every possible combination to design a product family with respect to the cost of the product family, i.e. cost-optimizing the commonality, which is technically possible

Now we know which commonalities are possible from a technical perspective. In contrast to Eichstetter et al. (2015), we do not look for the highest commonality possible, but for the cost-optimal degree of standardization. Therefore, we need quantitative cost models, which can depict: (1) manufacturing cost per unit, (2) cost effects related to the number of production/purchase units, (3) complexity cost to generate a new variant.

(1) typically increases with higher degree of standardization. (2) and (3) decrease with higher degree of standardization. Consequently, we need the number of each product produced. Figure 2 shows the algorithm used to determine the cost-optimal product family design. 


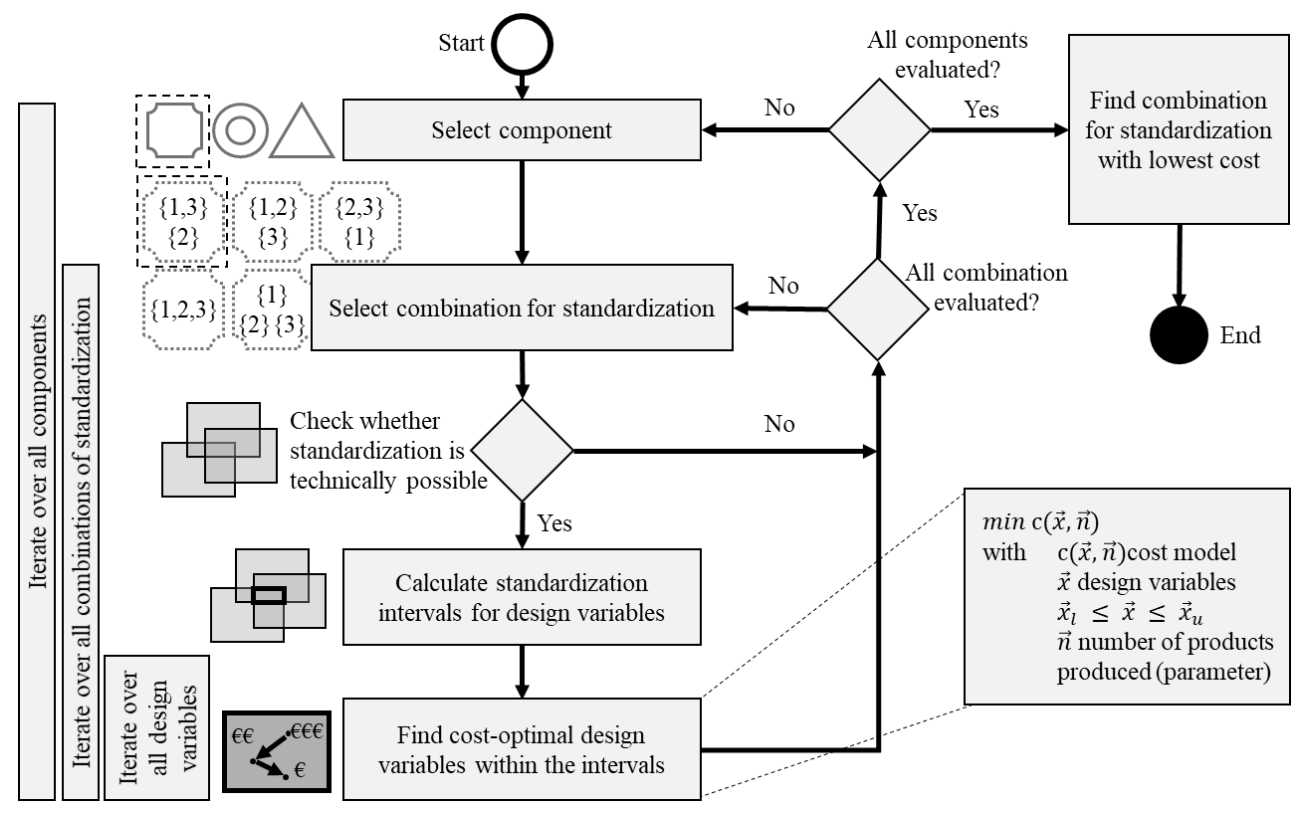

Figure 2. Algorithm to determine cost-optimal product family design

\section{Case study: Developing a family of hose boxes}

The case study is taken from a database of completed consultancy projects. A product family of hose boxes is chosen due to its comparably low complexity. It consists of five product variants that vary in the hose length that is inserted in the box. (it is made up of five product variants that are scaled by 5-meter steps of the hose length). Along with the hose length, the three cost-driving components: housing, spring, reel are also scaled to enable the creation of the product variants. The question in scope is the following: Is it possible to save cost by sharing some of the components between product variants?

In order to keep the modelling and computational effort low, a simplified mathematical model containing a total of ten design variables is used. Figure 3 shows the simplified build of the hose box and its design variables. Additionally, ten different objective quantities are modelled for the hose box, including e.g. the force for the insertion of the hose or the aspect ratio of the housing. For every variant of the hose box requirements for the objective quantities are stated. Also, for every design variable an upper and lower bound is derived from the project data.

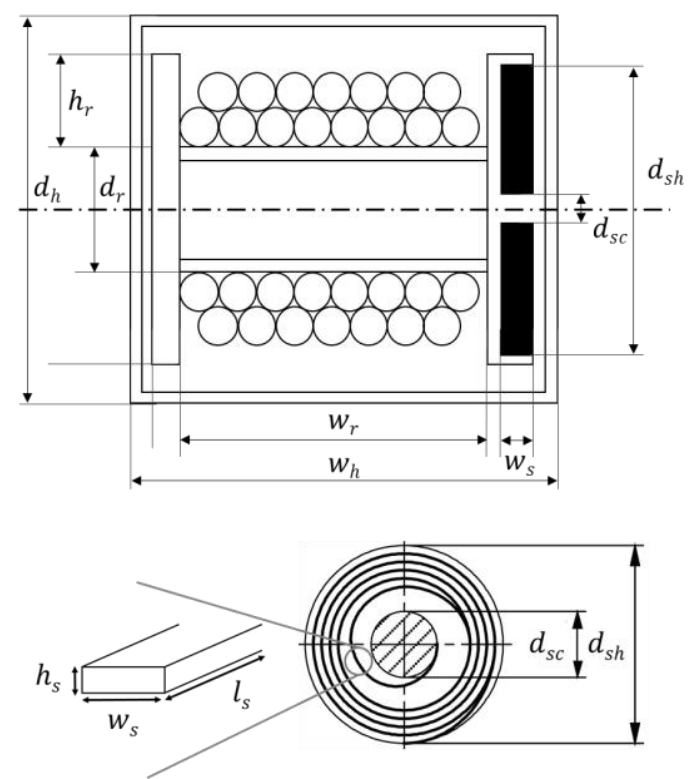

\begin{tabular}{|c|l|}
\hline Design Variable & \multicolumn{1}{c|}{ Description } \\
\hline$d_{r}$ & Outer diameter reel center \\
\hline$w_{r}$ & Inner width reel \\
\hline$h_{r}$ & Height reel jaw \\
\hline$w_{h}$ & Outer width housing \\
\hline$d_{h}$ & Outer diameter housing \\
\hline$h_{s}$ & Height spring strip \\
\hline$w_{s}$ & Width spring strip \\
\hline$l_{s}$ & Length spring strip \\
\hline$d_{s h}$ & Diameter spring housing \\
\hline$d_{s c}$ & Diameter spring core \\
\hline
\end{tabular}

Figure 3. Profile of the hose box and side view of the power spring 
With the completed mathematical model, we sequentially determine the solution spaces for every hose box variant. We use 100,000 samples to approximate the design space. For every sample point, the values of the objective quantities are calculated automatically and compared against the respective requirements. If no requirement is violated, the sample is valuated as a good design. To visualize the design space, it is projected onto five two-dimensional diagrams, depicting always two design variables. The five diagrams show all the same design space. Thus, the five diagrams are coupled with each other and change when borders of the design space are moved. The applied design projection tool draws good samples as green points and bad design points in another colour, referring to the objective quantity that is violated by the sample. Subsequently, a rectangular box is drawn in every diagram including as many as possible green points. Then, the boundaries of this box are modified iteratively until the box in every diagram includes only green dots. This box constitutes the solution space. Figure 4 shows the 2D-depiction of the determined solution space for the largest hose box. Following, the bounds of the objective quantities are changed to the values of the next hose box variant. We then modify the edges of the rectangular box until a satisfying box exists. It is important to notice that there are many different options to choose a rectangular solution space for a given system. The result therefore depends on the subjective criteria that are applied while determining the box. We apply two criteria:

1. Overlapping of the boxes

2. Prefer design variables values, which represent lower cost

The first criterium means choosing rectangular solution spaces for the different hose boxes in a way that the solution spaces overlap and hence enable commonality. Second, we prefer the regions of the solution space in which the values of design variables are low. This makes sense because the cost of the hose box correlates with the size of the components. Lower design variable values lead to lower cost.
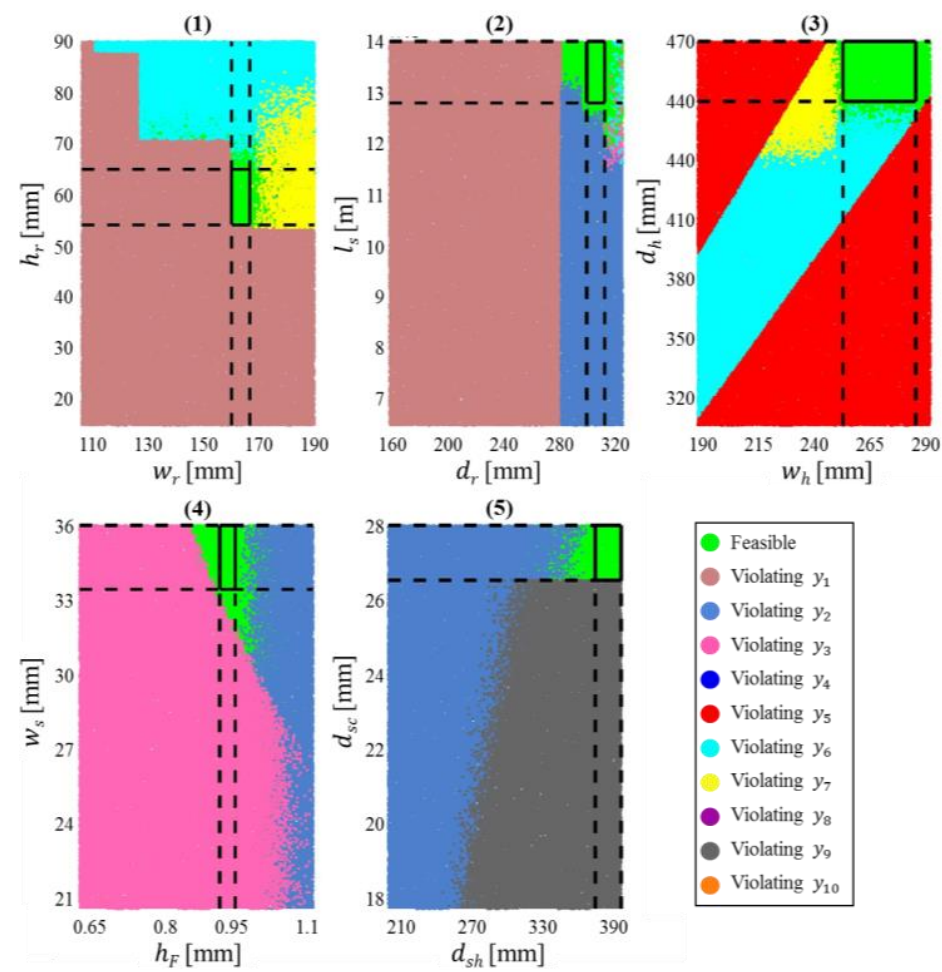

Figure 4. Determined solution space for the product variant: largest hose box

When a solution space for every product variant exists, the optimization of the product family starts. Figure 5 includes the solution space of all product variants for the three design variables for the costoptimal configuration of the product family. The grey bars represent the rectangular solution space for each variant. There are different options to choose common variable values between the product variant. A cost model makes it possible to evaluate, which of the options presents the optimal solution for the product family. 


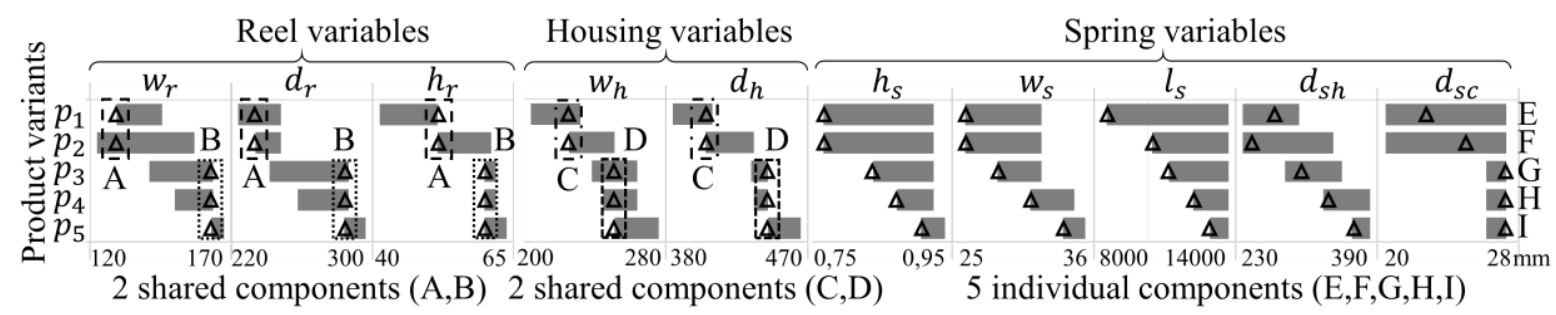

Figure 5. Comparison of solution spaces for the products and cost optimal variable values for the cost-optimal product family of hose boxes consisting of 9 components $(A-I)$

The applied cost model for the product family consists of three parts:

1. Material cost dependent on design variable values

2. Discount dependent on purchase quantity

3. Complexity cost dependent on number of components

As the components are purchased parts, no information about the production cost are available. Therefore, we assume that modelling the material cost is a sufficient consideration of manufacturing cost. Material cost are determined by multiplying a material cost factor with a cost driver that is calculated through the design variable values of the component. The cost of the housing for example is considered by a material cost factor that is stated as $€$ per $\mathrm{mm}^{2}$ and the cost driver which is given by the housing surface.

When components can be reused across product variants quantity increases, which potentially yields purchasing discounts. Relate the purchasing discounts to the concept of experience curves by the Boston Consulting Group (Henderson, 1973), in which costs decrease by a certain percentage for every doubling of the production volume. By analysing cost structures of an industry company they observed that a similar behaviour exists when relating the purchasing price with the purchasing volume. Therefore, we apply the concept of experience curve to account for a purchasing discount dependent on the quantity of the components. It is important to mention that the discussed cost effect only occur if a complete component is reused, that is, all design variables of the component need to be common. Complexity cost for the hose box include tooling cost that would incur for every additional component variant and an estimation of cost that arise for the administration of an additional variant. The optimization problem formulates as follows:

$$
\begin{aligned}
& \min _{\vec{x}} c(\vec{x}, \vec{n}) \\
& \text { with: } \vec{x}_{l} \leq \vec{x} \leq \vec{x}_{u} \text { : design variables of the products; } \vec{n} \text { : number of products produced (parameter) }
\end{aligned}
$$

$\vec{x}$ constitutes the design vector while $\vec{x}_{l}, \vec{x}_{u}$ are the lower and upper bounds of the overlapping solution boxes. $\vec{n}$ is the vector of the number of products produced and seen as a parameter. $c(\vec{x}, \vec{n})$ is the cost function that is to be minimized changing the design variables $\vec{x}$. A particle swarm algorithm is applied to solve the optimization problem for every different option of sharing the variables between subsets of the product variants.

As stated before, there are various options to share the components between product variants. According to the mathematical concept of Bell's number (Eichstetter et al., 2015) there are 52 theoretical possibilities to share a component between subsets of the 5 product variants. When combining the three components of the hose box there are $52^{3}=140608$ different possibilities to build the product family. To reduce the number of calculations we exclude all possibilities where components are shared only between not neighbouring products (e.g. a housing would be shared only between the smallest and largest product variant) because those options are not assumed to yield optimality. Figure 6 shows the cost of all calculated product families over the number of component variants that is used to build the product family. The cheapest product family results from using 9 component variants, composed by 2 reels, 2 housings and 5 power springs. The triangles in Figure 5 show the cost-optimal design variable values for reel, housing and spring. The results suggest that reel and housing should be shared by product 1 and 2 and by product 3, 4 and 5 respectively. The cost-sensitive spring should be optimized for each 
product variant separately. The approach provides both the cost-optimal combination of components within a product family and concrete numerical values to design those components.
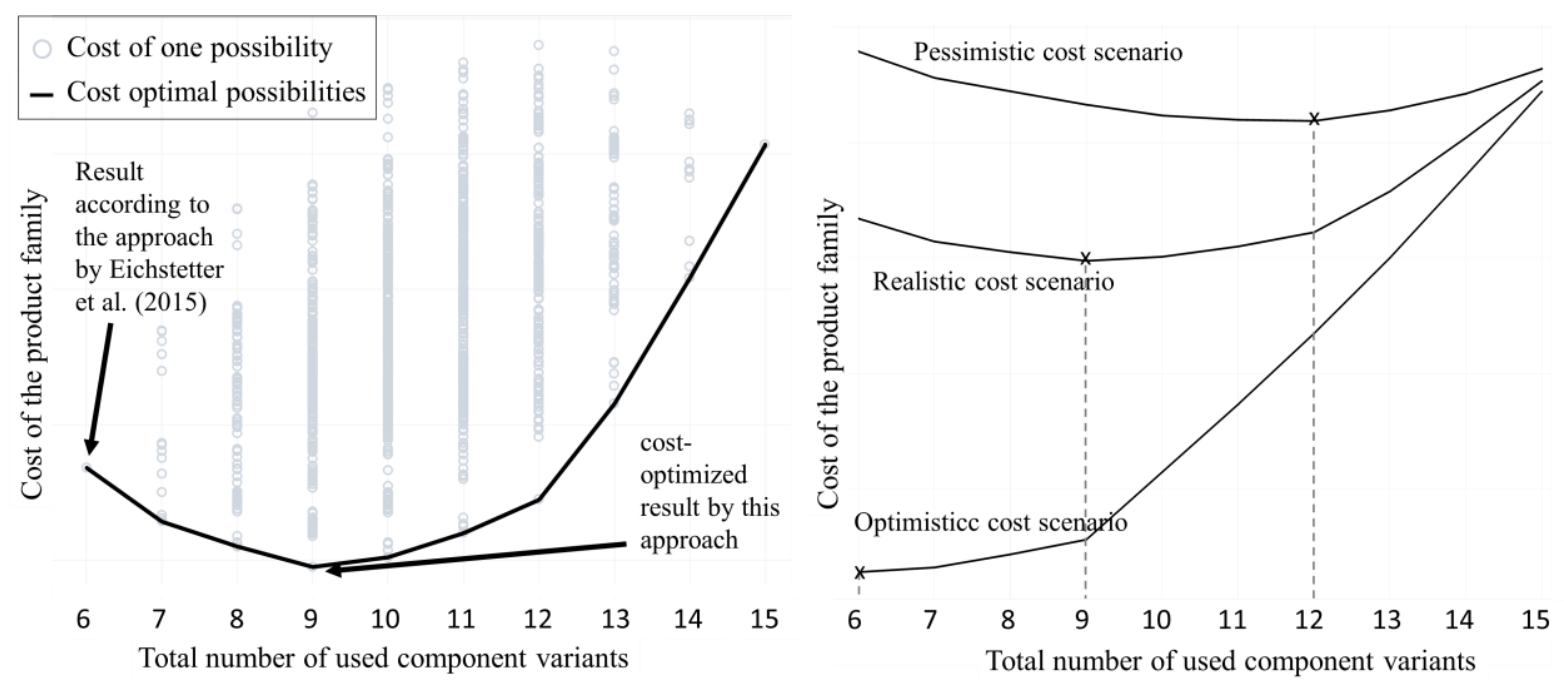

Figure 6. Cost of product family over the number of used component variants

Left: Cost of all possible combinations and cost-optimal curve Right: Impact of cost model on the result of the optimization

The applied cost model is based on assumptions. In order to reveal the influence of the chosen cost parameters on the results we present a sensitivity analysis in with a pessimistic and an optimistic scenario for the expected cost effects. The representative of the company categorized the scenarios according to his experience. Figure 6 reveals that the chosen cost parameters have a significant impact on the results. The more optimistic scenario shifts the optimum towards lower variety, while for the less optimistic scenario, the optimum can be found for a larger number of component variants.

\section{Summary and discussion}

In the beginning of this paper, we formulated the hypothesis that the highest degree of standardization is not always the best. Individual cost-optimal product design and design for commonality are often in conflict with each other. To find the cost-optimal product family design, we developed an approach existing of two main parts: (1) determining the possibilities for standardization from a technical perspective and (2) evaluating systematically the cost-optimal degree of standardization. For (1) we used existing approaches by Zimmermann et al. (2017) and Eichstetter et al. (2015). For (2) we used the results from (1) and cost models to optimize the design variables of our system.

We can show for a product family of hose boxes, that the optimal degree of standardization not always equals maximum commonality. It depends on the number of products produced, the production/purchasing costs and how much a new variant costs. All those parameters depend on both the product and the company. Therefore, we have developed a numerical approach, which allows the individual modelling of the product and its costs.

By decoupling the design variables using rectangular solution spaces, numerical effort can be reduced. The interaction between components does not need to be considered, which is especially helpful for complex products.

This leads to a drawback of this approach: By decoupling the design variables we lose solution space as we put a rectangular box in an arbitrarily shaped solution space. Good designs, which are outside of this box, are not considered. Thus, we might lose an overall optimal solution.

Furthermore, choosing the biggest solution space possible for each product does not necessarily turn into a higher possibility to find commonality between those products. 


\section{Outlook}

Although we have reduced the numerical effort by decoupling, still many evaluations are necessary to find an overall optimum. An increasing number of design variables leads to a higher number of system evaluations. More product variants increase combinatorics for standardization, which must be considered. Evaluations of the system model needed for Solution Space Engineering can be done in parallel, as well as the cost-optimization of the design variables. Thus, parallelization offers high potential to reduce computation time.

Although we applied the approach to a real-world problem, we cannot conclude whether this approach is suitable for industrial practice. Further application will be necessary to evaluate whether the benefits prevail the efforts, which are necessary to apply this approach. The user has to model the system of interest quantitatively, to be able to apply this approach. This causes high efforts. An intelligent database, which suggests suitable models to the user, might alleviate this problem.

Further research is necessary to analyse whether the loss of solution space due to decoupling is crucial for the global optimum. Therefore, the approach should be benchmarked with full optimization of the problem without decoupling. Different strategies on how to position rectangular solution space can be evaluated to define a process, which leads by experience to good results.

\section{References}

Chowdhury, S., Messac, A. and Khire, R. (2010), "Developing a Non-gradient Based Mixed-Discrete Optimization Approach for Comprehensive Product Platform Planning (CP3)", In 13th AIAA/ISSMO Multidisciplinary Analysis Optimization Conference, Fort Worth, Texas, American Institute of Aeronautics and Astronautics, Reston, Virigina, p. 583. https://doi.org/10.2514/6.2010-9174

Chowdhury, S., Messac, A. and Khire, R.A. (2011), "Comprehensive Product Platform Planning (CP3) Framework", Journal of Mechanical Design, Vol. 133 No. 10, p. 294. https://doi.org/10.1115/1.4004969

Eichstetter, M., Müller, S. and Zimmermann, M. (2015), "Product Family Design With Solution Spaces", Journal of Mechanical Design, Vol. 137 No. 12, https://doi.org/10.1115/1.4031637

Fellini, R. et al. (2004), “A sensitivity-based commonality strategy for family products of mild variation, with application to automotive body structures”, Structural and Multidisciplinary Optimization, Vol. 27 No. 1-2, pp. 89-96. https://doi.org/10.2514/6.2002-5610

Henderson, B. (1973), "The Experience Curve. Reviewed (Part II)".

Khajavirad, A. and Michalek, J.J. (2008), “A Decomposed Gradient-Based Approach for Generalized Platform Selection and Variant Design in Product Family Optimization", Structural and Multidisciplinary Optimization, Vol. 130 No. 7, pp. 1-8. https://doi.org/10.1115/1.2918906

Simpson, T.W. (2004), "Product platform design and customization: Status and promise", Artificial Intelligence for Engineering Design, Analysis and Manufacturing, Vol. 18 No. 1, pp. 3-20. https://doi.org/10.1017/ s0890060404040028

Simpson, T.W. (2006), "Methods for Optimizing Product Platforms and Product Families", In: Simpson, T.W., Siddique, Z. and Jiao, J. (Eds.), Product platform and product family design: Methods and applications, Springer, New York. https://doi.org/10.1007/0-387-29197-0_8

Simpson, T.W. and D'Souza, B.S. (2004), "Assessing Variable Levels of Platform Commonality Within a Product Family Using a Multiobjective Genetic Algorithm”, Concurrent Engineering, Vol. 12 No. 2, pp. 119-129. https://doi.org/10.1177/1063293x04044383

Simpson, T.W., Maier, J.R. and Mistree, F. (2001), "Product platform design: method and application", Research in Engineering Design, Vol. 13 No. 1, pp. 2-22. https://doi.org/10.1007/s001630100002

Skirde, H., Kersten, W. and Möller, K. (2015), Kostenorientierte Bewertung modularer Produktarchitekturen, Zugl.: Hamburg-Harburg, Techn. Univ., Institut für Logistik und Unternehmensführung, Diss., 2015, Reihe, Vol. 20 No. 1. Aufl., Eul, Lohmar.

Zapico, M. et al. (2015), "Towards product platform introduction: optimising commonality of components", in Weber, C., Husung, S., Cascini, G., Cantamessa, M. and Marjanović, D. (Eds.), The 20th International Conference on Engineering Design (ICED15): 27th-30th July 2015, Politecnico di Milano, Italy proceedings of ICED15, Design Society, Glasgow, Scotland.

Zimmermann, M. et al. (2017), "On the design of large systems subject to uncertainty", Journal of Engineering Design, Vol. 28 No. 4, pp. 233-254. https://doi.org/10.1080/09544828.2017.1303664 